http://dx.doi.org/10.18359/ravi.1706

\title{
Educación a distancia, interactiva y ubicua para el aprendizaje de lengua inglesa $^{1}$
}

\author{
Soraya García-Sánchez² \\ Universidad de Las Palmas de Gran Canaria, España
}

Recibido, julio 21 de 2015

Concepto evaluación, diciembre 31 de 2015

Aceptado, enero 27 de 2016
Referencia: García Sánchez, S. (2016). "Educación a distancia, interactiva y ubicua para el aprendizaje de lengua inglesa”. Revista Academia y Virtualidad, 9, (1), 68-88

\section{Resumen}

Este trabajo analiza el contexto de educación superior que permite el desarrollo de diversas tareas realizadas por un número de estudiantes universitarios con la intención de mejorar las competencias comunicativas de la lengua inglesa (Inglés como Lengua Extranjera -ILE- e Inglés para Fines Específicos - IFE-) a través de un sistema de educación a distancia (EaD), fundamentado en un enfoque de aprendizaje comunicativo, interactivo y ubicuo (u-Learning). Hay numerosas metodologías para el aprendizaje de lenguas extranjeras, a menudo favorecidas por el acceso a material digital contextualizado, según las necesidades de los estudiantes. Este caso de estudio muestra cómo la participación en distintas actividades (individuales y cooperativas), que requieren no sólo de la reinterpretación sino de la construcción del conocimiento, proporciona la mejora de las competencias comunicativas y del desarrollo de habilidades en un contexto de aprendizaje autónomo y cooperativo de educación superior a distancia.

Palabras clave: aprendizaje contextualizado, aprendizaje ubicuo, educación a distancia, enfoque comunicativo, ILE, IFE.

\footnotetext{
1 Artículo de Investigación, resultado de la línea del grupo de investigación Fletatis, Universidad de Las Palmas de Gran Canarias. España.

${ }^{2}$ Doctora en Filología Inglesa. Investigadora en el Grupo de Investigación FLETATIS, y profesora de lengua inglesa (ILE, IFE) y literatura-cultura anglosajona en la Universidad de Las Palmas de Gran Canaria, Islas Canarias, España. Correo: soraya.garcia@ulpgc.es
} 


\title{
Distance, interactive and ubiquitous learning of English language
}

\begin{abstract}
This paper analyzes the context of higher education that lets a development of several tasks performed by a number of college students in an attempt to improve communication skills in the English language (English as a Foreign Language -EFL- and English for Specific Purposes -ESP-) through a system of distance learning (DL) based on a communicative, interactive and ubiquitous learning approach (u-Learning). There are several methodologies for learning foreign languages, often favored by access to contextual digital material according to the students' needs. This study case shows how participation in various activities (individual and cooperative), which require not only the reinterpretation but knowledge construction, provides improved communication skills and developed skills in an environment of autonomous and cooperative learning of distance higher education.
\end{abstract}

Keywords: contextual learning, ubiquitous learning, distance learning, communicative approach, EFL, ESP.

Educação a distância, interativa e ubíqua para o aprendizado da língua inglesa

\section{Resumo}

Este trabalho analisa o contexto da educação superior que permite o desenvolvimento de diversas tarefas realizadas por um número de estudantes universitários com a finalidade de melhorar as competências comunicativas da língua inglesa (Inglês como Língua Estrangeira -ILE e Inglês para Fins Específicos -IFE) através de um sistema de educação a distância (EaD), fundamentado numa abordagem de aprendizado comunicativo, interativo e ubíquo (u-learning). Tem inúmeras metodologias para o aprendizado de línguas estrangeiras, frequentemente favorecidas pelo aceso a material digital contextualizado, segundo as necessidades dos estudantes. Este estudo de caso mostra como a participação nas diferentes atividades (individuais e cooperativas), que requerem não só da interpretação senão da construção do conhecimento, permite o melhoramento das competências comunicativas e do desenvolvimento de habilidades num contexto de aprendizado autónomo e cooperativo de educação superior à distância.

Palavras chave: aprendizado contextualizado, aprendizado ubíquo, educação à distância, abordagem comunicativa, ILE, IFE. 


\section{Introducción}

La lengua inglesa, ya sea en un contexto de aprendizaje general (Inglés como Lengua Extranjera - IFE), que permita la comunicación verbal y escrita, como en un contexto específico (Inglés para Fines Específicos - IFE), resulta necesaria utilidad en la actual comunidad de estudiantes universitarios que se forma constantemente para optar a mejores ofertas laborales. Las universidades españolas se han visto forzadas a transformar sus programas de estudio para adaptarse al propósito del Espacio Europeo de Educación Superior (EEES). Por ello, la evaluación de los contenidos se realiza a través de tareas que demuestren las adecuadas competencias que se plantean en los proyectos docentes de las asignaturas en cuestión. Los estudiantes, gradualmente, han pasado a ser los participantes activos y protagonistas de sus propias acciones de aprendizaje. El profesor se ha puesto en un segundo plano como instructor, y sobre todo, como guía de este proceso (Martínez y Amaro, 2008). A su vez, se requiere que los estudiantes -que acceden a los nuevos grados de las universidades canarias- desarrollen competencias de autonomía y cooperación en su primer año en Educación Superior (Martín-Santana y García-Sánchez, 2014), para que así consigan acreditar su nivel B1 (usuario independiente o nivel umbral) en una lengua extranjera al finalizar su titulación. Es indudable que la preferencia "obsesiva", como han calificado Jarabo Friedrich y García Álvarez (2014), suele ser el idioma inglés que se ha impuesto de repente en la comunidad educativa (MCERL, 2002). Quizás esa insistencia en el reconocimiento de la habilidad lingüística en lengua inglesa, que permita una comunicación adecuada en entornos diversos, se debe a su repercusión global y, por consiguiente, a su necesaria inserción en el mercado laboral más competitivo. Como apunta Torres Menárguez (2014), lo importante no es aportar certificaciones sino el dominio del idioma a nivel europeo (Cambridge English Assessment) o a nivel internacional con exámenes de reconocimiento internacional como el TOEFL (Test of English as a Foreign Language) o el IELTS (International English Language Testing System).

La Universidad de Las Palmas de Gran Canaria (ULPGC) ha actualizado sus licenciaturas a grados adaptados al EEES. Éstos pretenden responder a las necesidades de la vigente población. Entre sus objetivos se propone que los futuros graduados realicen al menos doce de sus créditos en una lengua extranjera, la cual suele ser la lengua inglesa. Se requiere que todo estudiante de grado demuestre que tiene el nivel B1 de competencia lingüística, bien sea aprobando las asignaturas de la titulación destinadas para dichos fines, o bien sea mostrando la acreditación lingüística a través de una institución oficial externa.

En la ULPGC, el Grado en Ingeniería en Telecomunicaciones, por ejemplo, ha desarrollado un diseño apropiado al ofrecer asignaturas que, por un lado, ponen al estudiante en el escenario adecuado para desarrollar competencias generales comunicativas en lengua inglesa, con la asignatura "Competencias comunicativas en inglés" (ILE), y por otro, mejoran la expresión oral y escrita en lengua inglesa en un contexto específico y profesional para los ingenieros de telecomunicaciones con la asignatura obligatoria de tercero, "Inglés" (IFE). La superación de ambas asignaturas obligatorias supone la adquisición del nivel de competencia B1 del MCERL que se requiere al concluir este grado. Como muestra Halbach et al., en esta adaptación "de las universidades españolas a la acreditación de lengua inglesa prevalece la diversidad y la divergencia", y resulta lógico que se sigan revisando las titulaciones para mejorarlas prestando atención a la sociedad que la integra y así llegar a un aceptación unísona (Halbach, 2013:8).

En la ULPGC existen, por un lado, titulaciones fundadas en entornos de aprendizaje presencial, que a su vez se apoyan de distintas herramientas TIC para su propósito (b-Learning), y por otro, entornos de aprendizaje a distancia que se 
establecen en la estructura de teleformación. La educación a distancia (EaD) más innovadora se caracteriza por el acceso y uso de recursos digitales móviles y ubicuos que facilita al aprendiz acceder constantemente a entornos de aprendizaje actualizados y participativos. En ambos casos, el modelo de plataforma que utilizan estos estudiantes de $\mathrm{EaD}$ de grado y postgrado es Moodle.

Este trabajo se contextualiza en la estructura de teleformación, en las asignaturas obligatorias en lengua inglesa "Comunicación oral y escrita para el desarrollo profesional" (EFL o ILE) e "Inglés aplicado a trabajo social" (ESP o IFE) para intentar mostrar cómo se hace necesaria una personalización del aprendizaje en espacios virtuales de aprendizaje a distancia. La educación a distancia es cada vez más participativa y cooperativa gracias a los recursos digitales que ayudan a mejorar las competencias lingüísticas en lengua inglesa. Al mismo tiempo, esta propuesta pretende incentivar la mejora de la formación continua al promover el aprendizaje creativo, ya sea compartido o individual, formal o informal. Para ello, este artículo propone reflexionar sobre metodologías actuales de docenciaaprendizaje, y dar respuesta a las siguientes cuestiones:

1. ¿Facilita la estructura de teleformación de la ULPGC la creación de un aprendizaje contextualizado por y para los estudiantes en lengua inglesa?

2. ¿Pueden los estudiantes universitarios mejorar sus competencias lingüísticas en lengua inglesa a distancia a través de un método de aprendizaje a distancia y ubicuo?

3. ¿Qué herramientas y tareas invitan al aprendizaje creativo y a la interacción de los estudiantes?

\section{Antecedentes y literatura}

A través de distintas metodologías de aprendizaje a las que puede acceder el estudiante de ILE o de IFE, atendemos como docentes a diversidad de inteligencias y formas de aprendizaje (Gardner, 1993). El aprendiz es, sin duda, el protagonista que puede seleccionar su espacio de aprendizaje telepresencial, de teleformación o a distancia. Trabajará de forma individual o en grupo para realizar tareas que mejoren las competencias lingüísticas o las competencias transversales que integran las habilidades sociales, digitales y comunicativas para aprender aprendiendo. A su vez, la realización de actividades que, por un lado, guíen a los estudiantes al descubrimiento de las estructuras de la segunda lengua (L2) o que, por el otro, faciliten la comprensión de textos cuyo léxico es innovador para el alumno forma parte de estrategias innovadoras de aprendizaje centradas en el aprendiz (Kalantzis y Cope, 2012).

En la actualidad, muchos docentes consideran que el aprendizaje es flexible en tiempo y espacio (ubiquitous learning) para estudiantes que acceden constantemente a la red no solo para buscar información, sino para realizar tareas específicas y socializarse (Cope y Kalantzis, 2010; Fernández Gómez et al., 2009). Aunque el aprendizaje ubicuo suele apoyarse de la propia red, también se manifiesta para que suceda fuera del aula y fuera del horario limitado de las instituciones académicas (Darias-Marrero, García-Sánchez y Vidal-Luengo, 2013; Hwang, Tsai y Yang, 2008, Kalantzis y Cope, 2012). El aprendizaje ubicuo ha sustituido la sala de fotocopias, proporcionando a los alumnos con el acceso y/o descarga de material informático en sus ordenadores, portátiles, móviles o tabletas, por ejemplo. Además los recursos no solo se descargan sino se reescriben facilitando a los estudiantes más exigentes crear sus propios espacios de aprendizaje compartido. Por ello, a través del aprendizaje móvil (m-learning) cada vez se hace más reiterativo el uso de dispositivos móviles que acompañan al usuario en todo momento favoreciendo un aprendizaje en constante movimiento (García-Sánchez, 2012; Sánchez Prieto, Olmos Migueláñez, y 
García-Peñalvo, 2014). De una u otra manera, el estudiante cuenta con una inmensa cantidad de información, que a veces es guiada y formal $\mathrm{y}$, otras veces, descubierta de forma autónoma e informal, a través de cualquier evento, noticia o plataforma digital. En este caso, el alumno está aprendiendo día a día no solo a estructurar sino a reconocer su plan de aprendizaje (personalised learning). Como apuntó Redecker et al. (2011), aunque estemos acostumbrados a mencionar los conceptos de la "personalización", la "cooperación", y la "informalización", el proceso de aprendizaje de los futuros ciudadanos depende de la combinación de los mismos (9).

La educación a distancia nace de la necesidad de instrucción y retroalimentación entre un maestro y sus discípulos que están separados por largos recorridos que no permiten la comunicación cara a cara. Varios investigadores han señalado que desde el siglo XII las cartas se utilizaron como medio de comunicación instructivos entre el docente y el alumnado que vivía incluso en otros países y continentes (Alfonso Sánchez, 2003: 4; Bravo Reyes, 1999: 59; Collazo Delgado, 2004: 14). Actualmente la educación a distancia se ha beneficiado de la integración de los estudiantes en una comunidad de aprendizaje virtual, y por ello, ha evolucionado en sus distintas etapas a través de la correspondencia, la telecomunicación y la telemática (García Aretio, 1986, 1999). Hoy, podríamos añadir que estamos en un periodo caracterizado por la movilidad, la flexibilidad, la colaboración entre los participantes de la comunidad educativa y por la ubicuidad a la hora de acceder y elaborar las tareas programadas para el curso a distancia. Los actuales usuarios disponen de dispositivos móviles que les permiten acceder al conocimiento desde cualquier espacio y a cualquier hora. Además, la reciente web 4.0 se define por su carácter ubicuo al permitir a la comunidad educativa-social estar hiperconectada con tecnologías cambiantes que sirven como vehículo de intercomunicación para formar una web íntegra (Vizoso: 243). Por ello, podríamos añadir a la clasificación de García Aretio (2014) que la educación a distancia actual podría caracterizarse por la ubicuidad que agiliza la mejor adaptación de los estudiantes al aprendizaje permanente a lo largo de sus vidas.

Según Kalantzis y Cope (2006, 2012), estamos ante una nueva forma de aprendizaje (New Learning), en la cual los estudiantes se convierten en diseñadores activos o codiseñadores de su propio aprendizaje. El docente no impone un único plan de aprendizaje sino que prepara una diversidad de tareas a la hora de aprender. Existen mayores oportunidades para la creación de contenido por parte del aprendiz. Las restricciones singulares de impartición de contenidos han dado paso a la pluralidad, y con ello, a la autonomía para generar material que se reinterpreta constantemente. Esto no es lo novedoso pero sí la actuación directa del estudiante en su proceso de construcción del conocimiento que, a su vez, debe fundamentar. El enfoque New Learning se centra en el aprendiz de hoy que ya no es solo nativo digital (Prensky 2001a y b) sino residente digital (White y Le Cornu, 2011). El estudiante actual además de querer implicarse de forma activa en su propio proceso de aprendizaje, también convive con la era virtual y las redes sociales. Así, el profesor (inmigrante digital, Prensky 2001a y b, o visitante digital, White y Le Cornu, 2011) se actualiza constantemente para proporcionar al alumno espacios educativos que le permitan crear, diseñar, participar, exponer, explicarse, presentar contenido, colaborar en grupos, grabar un diálogo en parejas, debatir sobre temas de actualidad en el foro virtual de la asignatura y escuchar un mensaje e interpretarlo, entre otras tareas. Como apuntaban Gisbert y Esteve (2011), algunos aprendices del nuevo milenio (New Millenium Learners-NML), ya sean nativos o residentes digitales:

[L]legan a la universidad con una cierta alfabetización digital, ya que conocen algunas herramientas TIC y las saben utilizar, pero siguen sin tener adquiridas las competencias 
necesarias que les permitan, además, aplicar esta alfabetización y el dominio de estas herramientas en un contexto educativo $\mathrm{y}$, en concreto, en su proceso de formación para el aprendizaje (53).

Es decir, una cuestión es usar digitalmente los recursos y otro asunto es saberlos aplicar al contexto educativo y al propio proceso de aprendizaje. Indistintamente, el enfoque comunicativo (communicative approach) o enseñanza comunicativa de la lengua tiene como objetivo central el éxito de la comunicación, ya sea verbal o escrita, entre emisores y receptores en un contexto real de la Lengua Extranjera (LE). La estructura de teleformación de la ULPGC debería facilitar el desarrollo de competencias lingüísticas y comunicativas a través de herramientas de interacción asincrónica como las actividades auditivas y los foros de debate, y de interacción simultánea como en las salas de chat y Skype. Por ello, la utilización de grabaciones y materiales auténticos hace que el estudiante pueda aproximarse con mayor éxito al contexto real de la lengua inglesa. Algunos investigadores han demostrado que la motivación de los aprendices aumenta cuando intentan comunicarse (a través de contenido léxico y gramatical) con otros compañeros o con sus profesores hasta el punto de querer crear una nueva identidad en la LE. Dörneyei (2009) sugiere que la integración que ya definió H. Gardner es el deseo de aprender una lengua extranjera en una comunidad para comunicarse con sus miembros hasta llegar a convertirse en uno de ellos (2009: 22). El objetivo del enfoque comunicativo, por tanto, participa en el satisfactorio desarrollo de la expresión oral y escrita en lengua extranjera (Gómez López, Solaz Portolés y Sanjosé López, 2014).

\section{Roles y recursos: diseño de aprendizaje}

El proceso de aprendizaje es diseñado por el docente de la estructura de Teleformación con el fin de contribuir en el desarrollo de los participantes hasta cumplir con las tareas y competencias de sus proyectos. El docente y estudiante de lengua inglesa en la Estructura de educación a distancia de Teleformación en la ULPGC debe partir de un enfoque participativo, cuyo fin último sea la comunicación. Hoy es posible que el estudiante esté inmerso en la lengua y en la cultura inglesa a través de métodos de aprendizaje no presenciales que se sustentan del acceso a Internet y a los medios de comunicación que están al alcance de todos.

Inicialmente, los recursos elaborados tanto para "Comunicación" como "Inglés aplicado" forman parte de un espacio formativo en la estructura de teleformación en la que el estudiante dispone de contenido digital seleccionado por el equipo docente en diversos formatos: Vídeos, Word, PDF o PowerPoint. A su vez, la plataforma también dispone de diversos enlaces externos y recursos audiovisuales abiertos y gratuitos que no solo complementan el curso sino que ayudan a contextualizar el mismo a las necesidades de los presentes estudiantes. Por ello, estos recursos muy frecuentes en los cursos abiertos masivos en línea (MOOC, por su sigla original de Massive Open Online Courses) varían según el curso y el estudiante en cuestión. 


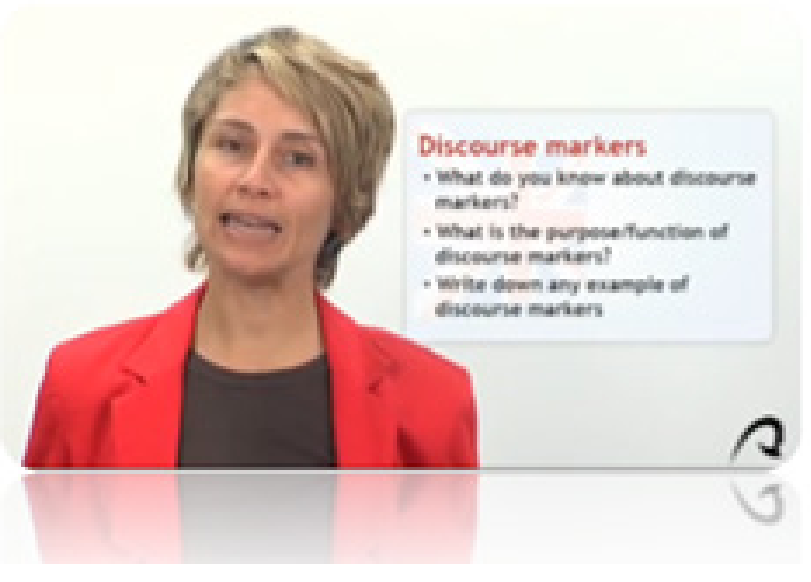

Figura 1. Ejemplo de vídeo explicativo creado en el proyecto de innovación educativa Prometeo/OCW en la ULPGC.

En segundo lugar, los estudiantes deben realizar un número de actividades digitales para superar el $50 \%$ de su evaluación continua y participativa. El otro $50 \%$ corresponde al examen final, que se desarrolla presencialmente en formato tipo test. Para la evaluación de la expresión oral, por ejemplo, ambos grupos de "Comunicación" e "Inglés aplicado" tenían que realizar un vídeo para exponer el contenido de manera oral, utilizando apoyo visual y digital. En "Comunicación" tenían que ver un documento audiovisual, y seguidamente, responder a unas cuestiones de comprensión auditiva para finalmente expresar su propia opinión sobre el tema del recurso audiovisual. En el caso de "Inglés aplicado", los alumnos tenían primero que completar los espacios de un texto con la mejor opción posible y luego, debían leerlo en voz alta. Finalmente, concluían su exposición con una opinión sobre el tema estudiado, como puede verse en la siguiente imagen.

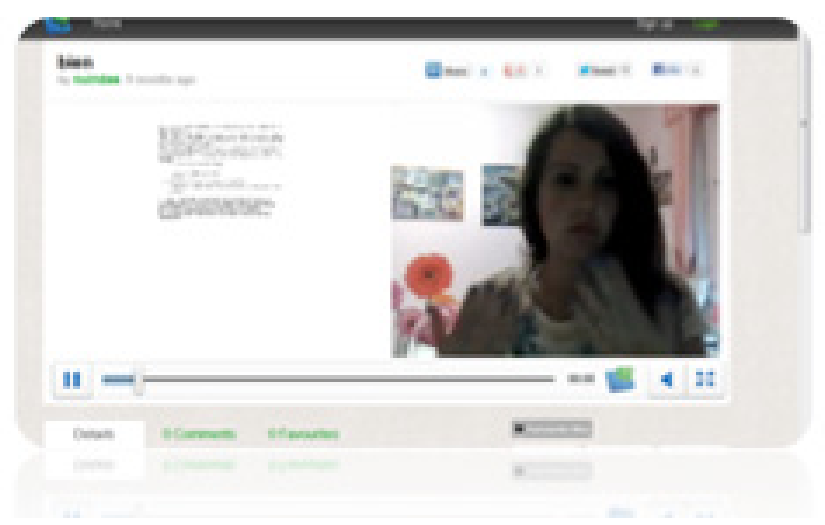

Figura 2. Ejemplo de presentación oral online utilizando las herramientas present.me y PowerPoint. 
Para la grabación de esta última actividad, el equipo docente de "Comunicación" e "Inglés aplicado" hizo lo posible por contextualizar la situación de aprendizaje y elaboró un vídeo explicativo haciendo uso de la herramienta que se sugería para la producción de sus propias grabaciones: present.me (Véase la Fig. 3). No se realizó ningún vídeo explicativo para YouTube porque ya había diversas reproducciones en este espacio que lo exponían. Por ello, se publicó el enlace del vídeo que según la valoración del equipo docente cumplía con una adecuada ilustración del proceso de grabación y edición de vídeos para que los estudiantes seleccionaran entre ambos recursos para sus videos presentaciones. En la mayoría de los casos, los estudiantes mostraron niveles de competencia digital elevados $(71 \%$ consiguieron realizar sin dudas posteriores). Se registraron, usaron su webcam o la cámara de sus teléfonos móviles o tabletas, utilizaron otros soportes digitales como fotos o diapositivas, añadieron música de fondo, accedieron a la grabación, y la publicaron (en su modalidad privada para present.me u oculta para YouTube) sin mostrar ningún tipo de dificultad. Aunque el $84 \%$ de estudiantes de ambas asignaturas eligió present.me por su sencillo interfaz para añadir el apoyo visual en formato PowerPoint, hoy esta herramienta ya no facilita un número de grabaciones mensuales en su modalidad gratuita y se ha optado por ofrecer también el recurso Picasst para la tarea de expresión oral.

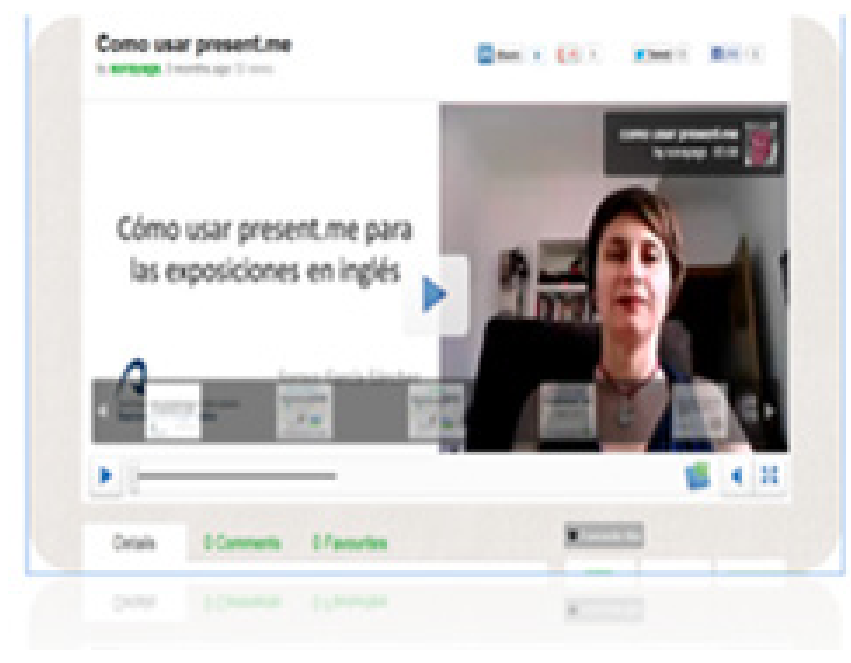

Figura 3. Pantallazo de vídeo explicativo del equipo docente para el uso de present. me.

\section{Diseño metodológico}

Este estudio se diseña a través de un proceso cuantitativo-cualitativo comparativo mediante observación, descripción y evaluación de resultados de dos asignaturas obligatorias en lengua inglesa que se imparten en la estructura de teleformación. Se analizan, a su vez, las tareas realizadas y la participación de los estudiantes en los foros de debate a distancia, así como el cuestionario realizado al final del curso con el fin de conocer sus valoraciones e inquietudes generales con respecto a las asignaturas cursadas (Véase el anexo). En este sentido, el cuestionario anónimo aportará datos significativos para una asignatura general de 
inglés comunicativo (ILE), "Comunicación oral y escrita para el desarrollo profesional" ("Comunicación" de aquí en adelante) y para una asignatura de inglés para fines específicos (IFE), "Inglés aplicado a Trabajo social" ("Inglés aplicado" de aquí en adelante). En ambas asignaturas el nivel de competencia lingüística es intermedio-B1 del MCERL (Marco Común Europeo de Referencia para las Lenguas).

\section{Muestra e instrumentos}

Este estudio comparativo y descriptivo examina la participación de doscientos veinte estudiantes matriculados en las asignaturas a distancia "Comunicación" e "Inglés aplicado" en la estructura de teleformación de la ULPGC durante un curso académico. Las variables que se tendrán presentes serán tanto cuantitativas como cualitativas. El 81\% de los estudiantes que cursa estas asignaturas reside en Gran Canaria, seguido de un número de estudiantes que vive en las otras Islas Canarias o en distintas ciudades del resto de España (Murcia, Madrid, Barcelona...) El número de estudiantes matriculados en la asignatura obligatoria de primer curso, "Comunicación", es de doscientos estudiantes, mientras que el número de estudiantes matriculados en la asignatura obligatoria de tercero, "Inglés aplicado" es de cuarenta estudiantes. Por un lado, se valorará los datos de una asignatura a distancia de inglés comunicativo general (ILE) y por otro, de una asignatura a distancia de un inglés específico (IFE), el de trabajo social.

Según el cuestionario anónimo, se observa cómo el $72 \%$ de los estudiantes lo forman mujeres mientras que el $28 \%$ lo constituyen hombres. Al tratarse de Educación Superior todos son mayores de 18 años y el 93\% se conecta a los cursos diariamente o al menos, más de tres veces por semana $(7 \%)$. Todos los estudiantes utilizan internet usualmente aunque no todos poseen de entrada las competencias digitales para crear documentos en formato audiovisual (43\% sí; 57\% no).

\section{Procedimiento}

Tanto en la asignatura "Comunicación" como en "Inglés aplicado", los estudiantes reciben el manual de la asignatura por correo postal, si no pueden recogerlo durante la primera sesión presencial. Los libros de texto elaborados para estas asignaturas en lengua inglesa tienen seis unidades. Están compuestos de lecturas, explicaciones de contenido léxico, gramatical y estructural, sus correspondientes ejercicios con respuestas, una propuesta de actividad digital ubicua, las palabras claves, la autoevaluación y las respuestas de los ejercicios planteados en cada unidad.

Para el plan de aprendizaje de "Comunicación" y de "Inglés aplicado" se sugiere que el estudiante dedique unas dos semanas a la lectura y adquisición de los contenidos de cada unidad de aprendizaje para que pueda realizar los ejercicios del libro y reflexionar sobre su aprendizaje. Finalmente, una vez se ha cumplido con las lecturas y ejercicios de autoevaluación, el estudiante debe formalizar distintas tareas digitales que comprenden contenido y actividades relacionadas con las unidades previamente estudiadas. Estas tareas, diseñadas por el equipo docente de la asignatura, están publicadas desde el comienzo del semestre en la estructura de teleformación a la que deben acceder para conocer las instrucciones. Una vez realizadas las actividades digitales, deberán subirlas a la aplicación telemática en un periodo máximo de dos semanas. Para la realización de tareas en "Comunicación" e "Inglés aplicado" las herramientas digitales más comunes son los documentos en formato Word, PowerPoint, vídeos en contexto (especialmente de YouTube) y los enlaces definidos para la creación de exposiciones o vídeos con Prezi, present.me, Picasst y YouTube para las exposiciones orales.

Estas actividades digitales se diseñan para que el estudiante cree su propio contenido de aprendizaje en lengua inglesa. La intención final es ayudar al aprendiz a contextualizar 
su aprendizaje, a comunicarse y a promover la creatividad y la interacción entre los participantes de la estructura de teleformación. Aunque el número de participantes difiere de una asignatura a la otra, en ambos casos se implementa el mismo número de tareas y con diversidades en formato (PPT, Prezi, Word, video-presentación), procedimiento (3 tareas o mini proyectos para 6 unidades de curso) y resultados (comunicación oral y escrita).

\section{Resultados y discusión}

Los resultados se recogieron al finalizar el curso, a través de la observación de resultados, de las reflexiones planteadas en el foro del curso o en los diálogos privados de tutoría, y de la recogida de datos del cuestionario anónimo. Sin embargo, durante el propio proceso de aprendizaje se iba observando qué recursos y herramientas utilizaban con más frecuencia los estudiantes para interactuar, y cómo iban desarrollando sus tareas digitales.

La estructura de teleformación: aprendizaje contextualizado en lengua inglesa

La estructura de teleformación cuenta con un número de herramientas disponibles a través de la plataforma Moodle. El glosario en línea, el foro de debate, el diálogo de tutoría privada, el acceso a vídeos/presentaciones en PowerPoint o Prezi creados los docentes y el acceso a otros enlaces publicados en la plataforma para mejorar la práctica oral o escrita de la lengua inglesa han sido los recursos que ha utilizado con frecuencia (2-3 veces por semana) la mayoría de estudiantes $(90 \%)$. Cabe destacar en esta línea que el foro de debate y el glosario fueron sugeridos de manera opcional y no toda la comunidad educativa participó en los distintas temas propuestos o añadió una palabra a definir y su ejemplo en contexto para crear de manera cooperativa un recurso léxico en contexto para todos. El $70 \%$ de estudiantes de ambas asignaturas participó en la creación del glosario, y el $88 \%$ de los estudiantes participó en, al menos, un foro de debate. El $54 \%$ sí lo hizo en todos los temas propuestos por el docente y los propios estudiantes.

Un número notable de estudiantes $(68 \%)$ sí ha podido demostrar en el desarrollo de las tareas programadas en "Comunicación" e "Inglés aplicado", la creatividad de su contenido al añadir otros recursos audiovisuales a sus trabajos y las referencias bibliográficas. La creación de contenidos se ha realizado en la fase de producción (Chan Núñez, 2004) que se establece para que el estudiante plasme distintos ejercicios que le permitan comprender y desarrollar el contenido formativo de la asignatura en cuestión. En esta etapa en particular, los estudiantes de Teleformación crearon su propio aprendizaje al reinterpretar el contenido con una reflexión presentada en formato digital. Al mismo tiempo, los participantes realizaron distintos ejercicios que promovían la creatividad. Un ejemplo de ello es el ejercicio de una tarea que indicaba la creación de un párrafo de 3-5 oraciones, que además de incluir los conceptos de "child protection" "refugees", "aid", se expresara en dos tiempos verbales distintos (con modales, presente simple y continuo, etc.), Los estudiantes ya habían trabajado la estructura de un párrafo y cómo desarrollar un mensaje coherente en un breve párrafo. Con este ejemplo de ejercicio que complementa a varios en una misma tarea, el producto final del párrafo es único y diverso dependiendo de la actitud, aptitud y competencia lingüística y comunicativa del estudiante.

Otro ejemplo de herramienta digital que facilitó la creación de contenidos por parte de los estudiantes es el glosario de Moodle. Para "Comunicación" e "Inglés aplicado" es fundamental no solo contar con el libro de texto del curso, sino que el desarrollo de la adquisición de nuevos términos que se ponen en contexto con el trabajo elaborado por los propios estudiantes. Las instrucciones del glosario son simples y claras. Se solicita que todos sigan la misma estructura, es decir, los alumnos deben incluir la pronunciación, que pueden revisar en diccionarios online ya presentados en el curso, además de la 
definición y un ejemplo del término en contexto. Al finalizar el periodo de entrega para esta actividad, los estudiantes pueden disponer del glosario del grupo en formato impreso o en formato digital creados por la comunidad de estudiantes que ha participado en ese curso.

Asimismo, el foro de debate de "Comunicación" y de "Inglés aplicado" fue la herramienta más interactiva de los alumnos, que continuamente respondían a propuestas, inquietudes, dudas y otros temas de debate planteadas tanto por el docente como por el resto de compañeros. M.J.W. Thomas (2002) argumenta que el foro se ha convertido en un instrumento de aprendizaje común en los estudiantes, similar a material del curso o a las clases magistrales tradicionales (352). La revisión de contenidos a través de un libro o de un vídeo en YouTube es hoy tan usual en el proceso de aprendizaje de un estudiante a distancia como el foro de debate. En esta línea, el foro en un enfoque de aprendizaje a distancia y ubicuo se ha convertido en una herramienta imprescindible que facilita el acercamiento entre los estudiantes, el docente y la comunidad universitaria.

Como apunta Garay, Luján y Etxebarria (2013), las herramientas de la web 2.0 "contribuyen a desarrollar las estrategias sociales [y] se caracterizan por ser actividades para practicar lo que se está aprendiendo, para recopilar y para gestionar la información" (172). El foro en "Comunicación" e "Inglés aplicado" admite el desarrollo del discurso crítico en lengua inglesa, así como de la comprensión y la adecuada producción de lenguaje comunicativo en inglés. Según el cuestionario anónimo, el $88 \%$ de los informantes manifestaron que revisaban $\mathrm{su}(\mathrm{s})$ párrafo(s) antes de publicarlos en la plataforma, prestando atención a la estructura de sus oraciones y a la comprensión de sus mensajes. Esto indica que un elevado porcentaje de estudiantes mostró un nivel de esfuerzo alto o medio alto con respecto al aprendizaje de la lengua inglesa. Ya Rodríguez Juárez y Oxbrow (2010) señalaron que las razones que invitan a los estudiantes a participar en los foros de debate son la interacción entre los usuarios y la mejora de la competencia lingüística en lengua inglesa (150).

En este entorno de $\mathrm{EaD}$, la reflexión, el esfuerzo y la motivación por conseguir los objetivos del curso se desarrollaron en un contexto de aprendizaje autónomo y cooperativo. En esta línea, se propuso mejorar la expresión oral y escrita de sus participantes teniendo en cuenta la interacción y un número mínimo-máximo de palabras. Los datos del siguiente gráfico indican que los estudiantes están cada vez más acostumbrados a mostrarse públicamente y no solo a responder a las sugerencias planteadas por los docentes sino a iniciar ellos mismos temas de debate considerablemente interesantes. Como es lógico, si mayor es el número de estudiantes, mayor es el número de contribuciones al foro como sucede en la asignatura con mayor número de estudiantes, "Comunicación":

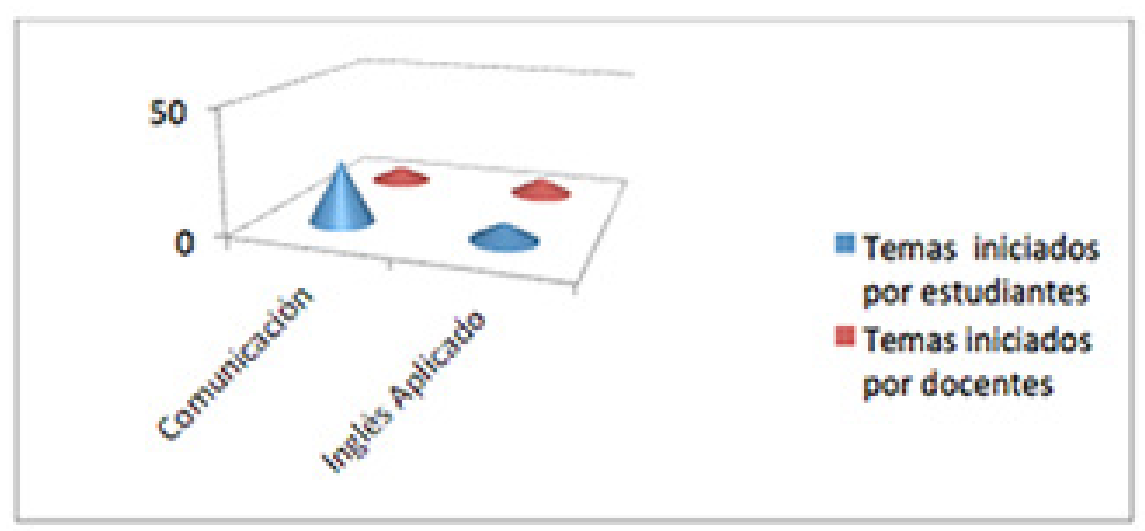

Gráfico 1. Iniciativa de los estudiantes en los foros de debate de "Comunicación" e "Inglés aplicado". 
En la asignatura "Comunicación" hubo 4 temas de los 31 que se abrieron en el foro del curso que revelaron un mayor nivel de participación e interacción entre los estudiantes. Tanto en "Comunicación" como en "Inglés aplicado", el tema más participativo fue "Introduce Yourself" (182 de los 240 estudiantes en ambas asignaturas) que se publicó al comienzo de curso con el fin de crear un ambiente de aprendizaje representativo de los estudiantes a distancia. En este foro de debate inicial se solicitó a los estudiantes una introducción en lengua inglesa similar en la estructura y número de palabras a la presentada por la docente. Con esta iniciativa se pretendía favorecer la confianza del aprendiz a la hora de crear un párrafo de presentación. Otro tema de debate que mostró un alto nivel de participación en ambas asignaturas es el que lleva el título de "(UN) Successful Communication" (146 de 240 estudiantes), en el que se invitaba a los estudiantes a reflexionar sobre situaciones de comunicación adecuada o incorrecta que no solo justificaban en lengua inglesa sino que, a su vez, ilustraban con una imagen.

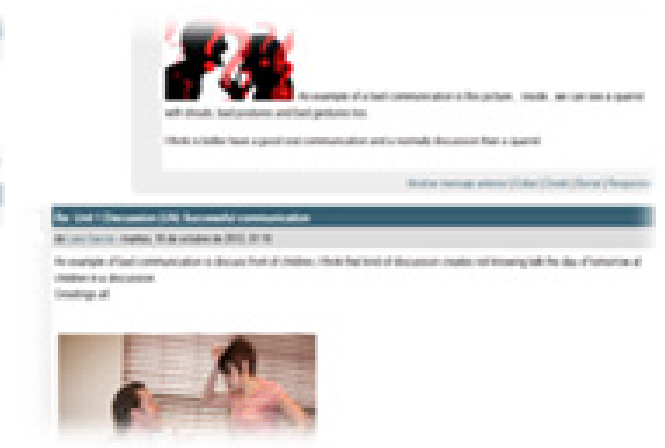

Figuras 4 y 5. Interacción argumentada sobre "comunicación” entre los estudiantes en "Inglés aplicado".

Mejora de competencias lingüísticas a través de educación a distancia ubicua

En línea con la creación de conocimiento según las distintas tareas digitales que debían completar, de menor a mayor grado, los estudiantes se sintieron especialmente alarmados por la realización de la tarea 3. Plantearon en el foro del curso diversas dudas con respecto al manejo de las herramientas digitales present.me o YouTube propuestas para la realización de la presentación-vídeo. La actividad 3 de "Comunicación" y de "Inglés aplicado" consistía en realizar una exposición oral en lengua inglesa utilizando un formato vídeo. Esto causó bastante incertidumbre entre algunos estudiantes de la estructura de teleformación que revelaron sus dudas al abrir distintos temas al respecto para conocer las sugerencias de la docente y de los propios compañeros. En esta línea, la herramienta del diálogo de tutoría privada también incrementó su uso demostrando que la competencia digital de estos alumnos de educación a distancia no parece ser tan elevada como podríamos esperar.

En cualquier caso, los estudiantes supieron adaptarse a los requisitos de la tarea 3 , y respondieron a la mejora de su competencia 
digital al formalizar el ejercicio de manera adecuada. Con respecto a las 3 tareas digitales de evaluación que los estudiantes de "Comunicación" e "Inglés aplicado" publicaron en la plataforma (Una por dos unidades de aprendizaje diseñadas a partir del manual del curso), es notable destacar que la mayoría de los alumnos demostraron la adquisición del contenido lingüístico y desarrollaron, por consiguiente, competencias comunicativas y digitales utilizando distintos documentos en formato Word o PowerPoint, además de vídeos que, en este último caso, capturaron no solo sus competencias lingüísticas y comunicativas sino su mejora en el lenguaje no verbal a través de una adecuada postura corporal y expresión facial (88\%).

Es indudable que el espacio de la interacción en "Comunicación" e "Inglés aplicado" ha permitido la mejora de competencias vinculadas a la expresión lingüística en un contexto de aprendizaje comunitario, optimizando con ello, la diversidad de opiniones. El ejemplo más usual de este tipo de entorno de aprendizaje interactivo ha sido el foro de debate que el docente había planificado para establecer una continuada interacción entre los propios estudiantes. En otras ocasiones, los alumnos abrieron nuevos temas que invitaban al diálogo o a la resolución de dudas. Las tutorías virtuales se presentan como otro ejemplo de espacio de interacción virtual, ya que los alumnos plantearon de forma privada las cuestiones que pudieran llegar a tener.

En esta línea ha sido esencial que los estudiantes de "Comunicación" e "Inglés aplicado" se esforzaran en desarrollar su comprensión lectora y auditiva, así como en plasmar mensajes a través de su expresión escrita en lengua inglesa. Igualmente, han podido optimizar sus competencias digitales a través de la elaboración de ejercicios en formato Word, PowerPoint, o en formato vídeo, la subida y envío de documentos a la plataforma y la participación en foros de debate, entre otras.
La educación a distancia desde un enfoque de aprendizaje ubicuo prepara a los participantes a la reflexión sobre su propio conocimiento creado a cualquier hora y desde cualquier lugar. Se fomenta, de esta manera, el desarrollo de competencias de aprendizaje autónomo (Gómez-López et al., 2014: 13-14). La educación a distancia puede considerarse de éxito si el participante reconoce su responsabilidad y compromiso a la hora de demostrar su aprendizaje.

En general, si se observan los datos del cuestionario y se valora la pregunta 14 en la que se les pedía sugerencias de mejora, el $87 \%$ de los alumnos indicó que incluiría más actividades de desarrollo de competencia lingüística y comunicativa tanto para la mejora del aprendizaje autónomo, como para la necesaria interacción con los estudiantes, ya sea de forma escrita o de forma oral, como se puede apreciar en la tabla inferior. En cada caso, se demanda mayor actividad y presencia en la red por parte de los alumnos. Como afirma Seely Brown (2005), los sistemas de aprendizaje más innovadores se caracterizan por la acción del estudiante, más que por la acción del docente que facilita los contenidos: "We now need a new approach to learningone characterized by a demand-pull rather than the traditional supply-push mode of building up an inventory of knowledge in students' heads" (Seely Brown, 2005: 26). En este sentido, los estudiantes de "Inglés aplicado" y de "Comunicación" demostraron que están integrados en la sociedad y en la educación actual que los hace partícipes de su propia mejora educativa, personal y profesional. Es a través de la puesta en práctica de los contenidos, y de la adecuada combinación entre estos y los resultados a conseguir como se mejoras la acciones de aprendizaje de los estudiantes (García-Sánchez, 2014).

Como pone de manifiesto Pérez Cañado (2013) el modelo de evaluación por competencias debe ser flexible y demostrar un uso correcto de las TIC. Por ello, se debe atender a la diversidad de inteligencias y estilos de aprendizaje, 
además de adaptarse a los profesionales del futuro (Gardner, 1983). En la siguiente tabla, se observan las sugerencias más demandadas por los estudiantes de "Comunicación" y de "Inglés aplicado" al responder a la última pregunta del cuestionario anónimo de este análisis. Según las sugerencias planteadas
(Véase la columna de la izquierda) por los encuestados, se ha añadido la correspondiente competencia más demandada por los participantes de la estructura de teleformación (Columna de la derecha).

Tabla 1. Combinación de sugerencias y competencias más demandadas por los estudiantes de $\mathrm{EaD}$.

\begin{tabular}{|l|l|}
\hline \multicolumn{2}{|c|}{ Estudiantes de "Comunicación"/“'Inglés aplicado": EFL/ESP } \\
\hline Sugerencias & Competencias más demandadas \\
\hline More listening activities & $\begin{array}{l}\text { Lingüística, comunicativa y de } \\
\text { aprendizaje individual }\end{array}$ \\
\hline More videos with Teacher's explanations & $\begin{array}{l}\text { Lingüística, comunicativa y de } \\
\text { aprendizaje individual }\end{array}$ \\
\hline Happy with platform and teleformación & Digital \\
\hline $\begin{array}{l}\text { Using free software and open sources to } \\
\text { ensure universal access }\end{array}$ & Digital \\
\hline Happy with learning and teaching & $\begin{array}{l}\text { Lingüística, comunicativa y de } \\
\text { aprendizaje cooperativo }\end{array}$ \\
\hline $\begin{array}{l}\text { More on present.me-communicative } \\
\text { competence }\end{array}$ & $\begin{array}{l}\text { Lingüística, comunicativa y de } \\
\text { aprendizaje cooperativo }\end{array}$ \\
\hline $\begin{array}{l}\text { More discussion forums to improve } \\
\text { writing }\end{array}$ & $\begin{array}{l}\text { Lingüística, comunicativa y de } \\
\text { aprendizaje cooperativo }\end{array}$ \\
\hline IT abilities to be improved by students & Digital \\
\hline $\begin{array}{l}\text { More oral activities + resource to } \\
\text { interact with others }\end{array}$ & $\begin{array}{l}\text { Lingüística, comunicativa y de } \\
\text { aprendizaje cooperativo }\end{array}$ \\
\hline More present.me or YouTube activities & $\begin{array}{l}\text { Lingüística, comunicativa y de } \\
\text { aprendizaje individual-cooperativo }\end{array}$ \\
\hline
\end{tabular}




\section{Conclusiones}

Esta investigación ha pretendido vincular aprendizaje ubicuo con educación a distancia en Educación Superior. El contexto específico se diseñó para dos asignaturas obligatorias, una enmarcada en ILE y la otra en IFE como parte del Grado en Educación Primaria (GED), por un lado, y del Grado en Trabajo Social (GTS), por el otro, en la Estructura de Educación a Distancia de Teleformación de la Universidad de Las Palmas de Gran Canaria (ULPGC). Foros de debate, glosarios en línea, chats y elaboración de vídeos o representaciones/ exposiciones orales son algunas de las herramientas utilizadas para los trabajos elaborados por estos estudiantes de ILE e IFE con el fin de contribuir en el aprendizaje de competencias comunicativas y lingüísticas, así como de contenido específico a la mejor hora y desde cualquier lugar (aprendizaje ubicuo) en el que estuvieran conectados a la estructura de teleformación.

La estructura de teleformación de la ULPGC dispone de un plan de aprendizaje virtual a distancia cuyo fin es la mejora del conocimiento del aprendiz a través de la realización de unas tareas y pruebas. Este accede, estudia y participa en este entorno de aprendizaje ubicuo en el lugar y hora que mejor le conviene, y probablemente, a través de diversos dispositivos móviles (aprendizaje móvil). En esta línea, los estudiantes de "Comunicación" e "Inglés aplicado", que ya participan en Grados adaptados al Espacio Europeo de Educación Superior (EEES), han demostrado con éxito la superación de competencias comunicativas y digitales, así como de aprendizaje autónomo, cooperativo y permanente, tan necesarios en estos entornos de educación a distancia, y como del mismo modo promueve el EEES (lifelong learning).

Este estudio centrado en la participación y creación de contenido para ILE (EFL) y para IFE (ESP) manifiesta que es posible mejorar competencias comunicativas en lengua inglesa si se establece una metodología de aprendizaje a distancia cuyo enfoque sea comunicativo, interactivo y ubicuo. Conjuntamente, es usual que los actuales estudiantes estén acostumbrados a acceder a la red para socializarse, formarse, contribuir y conseguir metas profesionales. Por ello, la autonomía y cooperación, tanto digital como ubicua, se describen como habilidades esenciales de las que debería disponer la comunidad de estudiantes de la estructura de teleformación de la ULPGC. Sin embargo, cabe destacar que el proceso de adaptación ha sido especialmente complejo para algunos estudiantes que han tenido que demostrar su evolución en competencias digitales, lingüísticas y comunicativas en lengua inglesa.

En cualquier caso, los entornos de aprendizaje virtuales a distancia que se utilizan en instituciones educativas públicas como la ULPGC son variados y dependen del tipo de asignatura y del número de estudiantes con las que cuente el curso para su gestión más eficaz. Como Álvarez (2012) defiende, la red ha permitido que la metodología de enseñanzaaprendizaje no tenga sólo un enfoque personal y autónomo (Personal Learning EnvirnomentPLE), sino social, cooperativo y colaborativo. Hoy se ofrecen cursos masivos abiertos online, MOOCs (Massive Open Online Courses), que se centran en una democratización de la educación a la que se puede acceder a cualquier hora y desde cualquier lugar (ubiquitous learning).

Bien sea un MOOC o un curso a distancia avalado por una institución académica, los entornos de aprendizaje virtuales se siguen diseñando desde un enfoque participativo que facilite al estudiante el descubrimiento del contenido objeto de estudio. El proceso de enseñanza-aprendizaje de una materia en concreto se debería programar prestando atención a los estudiantes y a sus necesidades, es decir, valorando los espacios de aprendizaje disponibles, el contenido, las tareas individuales y cooperativas, las competencias, las necesidades de cada uno y la evaluación (personalised learning). A su vez, el diseño 
de un entorno virtual de educación a distancia debe partir de la necesidad de formar a personas autónomas, curiosas y sociables. En este sentido, la ULPGC continúa reorganizando sus titulaciones, así como sus metodologías de enseñanza-aprendizaje vinculadas a recursos TIC tanto en su modalidad presencial como en su modalidad no presencial, formando a los estudiantes del siglo XXI con el propósito de que sean los creadores de su propio conocimiento y los participantes de su propio aprendizaje.

\section{Referencias}

Álvarez, D. (2013). "Entornos personales de aprendizaje: del aprendizaje autónomo ala educación edupunk". En: J. Bergman y M. Grané (coord.), La universidad en la nube, A universidade na nuvem, pp. 239-262. Barcelona, LMI. Collecció Transmedia XXI, Laboratori de Mitjans Interactius, Universidad de Barcelona. Recuperado de http://www.lmi. ub.es/transmedia21/pdf/6_universidadnube. pdf [Consultado el 15 de junio de 2013].

Alfonso Sánchez, I. (2003). "La educación a distancia”. En: Revista ACIMED, 1(1), 1-14. Recuperado de http://eprints.rclis.org/5122/1/ educacion.pdf [Consultado el 28 de septiembre de 2014].

Bravo Reyes, C. (1999). Un sistema multimedia para la preparación docente en medios de enseñanza, a través de un curso a distancia. Ciudad de La Habana: Instituto Superior Pedagógico.

Chan Núñez, M. E. (2004). "Entornos de aprendizaje digitales". En: Revista Digital Universitaria, 5(10), 1-26. Recuperado de http://www.revista.unam.mx/vol.5/num10/ art68/int68.htm [Consultado el 12 de enero de 2014].

Collazo Delgado, R. (2004). Una concepción teórico-metodológica para la producción de cursos a distancia basados en el uso de las tecnologías de la información y las comunicaciones. Ciudad de la Habana: CREA.

Cope, B. y Kalantzis, M. (Eds.) (2010). Ubiquitous Learning: Exploring the anywhere/ anytime possibilities for learning in the age of digital media. Urbana-Champaign: University of Illinois.

Darias-Marrero, A.; García-Sánchez, S.; y Vidal-Luengo, A. R. (2013). "Aprendizaje móvil, ubicuo y autónomo de lenguas extranjeras en la ULPGC". En: Cuadernos de innovación educativa, 1, 11-35.

Dörneyei, Z. (2009). Research Methods in Applied Linguistics. Oxford: Oxford University Press.

Fernández Gómez, E., et al. (2009). U-learning. El futuro está aquí. México: Ed. Alfaomega.

Garay, U.; Luján, C.; y Etxebarria, A. (2013). "El empleo de herramientas de la Web

2.0 para el desarrollo de estrategias cognitivas: un estudio comparativo". En: Porta Linguarum, 20, 169-186. Recuperado de http://www.ugr. es/ portalin/articulos/PL_numero20/11\%20 \%20Urtza.pdf [Consultado el 15 de mayo de 2014].

García Aretio, L. (1986). Educación superior a distancia: Análisis de su eficacia.

Badajoz: UNED-Mérida. (1999). "Historia de la educación

a distancia”. En: Revista

Iberoamericana de Educación a Distancia (RIED), 2(1), 11-40.

(2014). Bases, mediaciones y futuro de la educación a distancia en la sociedad digital. Madrid: Síntesis.

Gardner, H. (1993). Frames of Mind: The theory of multiple intelligences. New York: Basic Books. 
García-Sánchez, S. (2012). "English in class and on the go: Multimodal u-Learning". En: The Eurocall Review, 20(2), 94-102.

. (2014). "Knowledge Creation and Digital Collaboration in Higher Education". En: Collaborative Learning: Theory, Strategies and Educational Benefits, S. Rutherford (Ed.), pp.1-14. New York: Nova Science Publishers.

Gisbert, M. y Esteve, F. (2011). "Digital learners: la competencia digital de los estudiantes universitarios". En: La Cuestión Universitaria, 7, 48-59. Recuperado de http:// www.lacuestionuniversitaria.upm.es/web/ grafica/articulos/imgs_boletin_7/pdfs/LCU-76.pdf [Consultado el 28 de mayo de 2014].

Gómez López, Á.; Solaz Portolés, J.J.; y Sanjosé López, V. (2014). "Competencia en lengua inglesa de estudiantes universitarios españoles en el contexto del EEES: nivel de dominio lingüístico, estrategias metacognitivas y hábitos lectores". En: Revista de Educación, 363. Recuperado de http://www.revistaeducacion.educacion.es/ doi/363 175.pdf [Consultado el 18 de febrero de 2015].

Halbach, A.; Lázaro Lafuente, A.; y Pérez Guerra, J. (2013). "La lengua inglesa en la nueva universidad española del EEES". En: Revista de Educación, 362, 105-132. Recuperado de http://www.revistaeducacion. educacion.es/doi/362_154.pdf [Consultado el 11 de octubre de 2014].

Hwang, G.-J.; Tsai, C.-C. y Yang, S.J.H. (2008). "Criteria, Strategies and Research Issues of Context-Aware Ubiquitous Learning". En: Educational Technology and Society, 11(2), 81-91.

Jarabo Friedrich, F. E. y García Álvarez, F. J. (2014). "Competencias en la enseñanza universitaria: una autocrítica customizada". En: Libro de Actas de las I Jornadas Iberoamericanas de Innovación Educativa en el ámbito de las TIC, pp. 399-406. Las Palmas de Gran Canaria: Universidad de Las Palmas de Gran Canaria.

Kalantzis, M.; y Cope, B. (2006). "Changing Subjectivities, New Learning”. En:

Pedagogies: An International Journal, 1, 7-12.

Kalantzis, M.; y Cope, B. (2012). New learning: elements of a science of education.

Cambridge: Cambridge University Press, second edition.

(2002) Marco Común Europeo de Referencia para las Lenguas: Enseñanza,

Aprendizaje, Evaluación, Madrid, Ministerio de Educación, Cultura y Deporte - Instituto Cervantes - Editorial Anaya. Recuperado de http://cvc.cervantes.es/obref/marco/default. htm [Consultado el 29 de julio de 2013].

Martín-Santana, C.; y García-Sánchez, S. (2014). "Nivel B1: Autonomía y trabajo de los estudiantes de primer año de EFL". En: Libro de Actas de las I Jornadas Iberoamericanas de Innovación Educativa en el ámbito de las TIC, pp. 41-50. Las Palmas de Gran Canaria: Universidad de Las Palmas de Gran Canaria.

Martínez, A.B.; y Amaro, R.E. (2008). "El docente universitario y su espacio de formación. En: Fundamentación de una propuesta en Docencia Universitaria, 9(2-55), 53-80.

Pérez Cañado, M.L. (2013). "The Development of ICT Competence within Bologna-Adapted Language Degrees". En: The International HETL Review, 3(1). Recuperado de http://www.hetl.org/academicarticles/the-development-of-ict-competencewithin-bologna-adapted-language-degrees [Consultado el 9 de abril de 2014].

Prensky, M. (2001a). "Digital natives, digital immigrants". En: On the Horizon, 9(5).

Recuperado de http://www.marcprensky. com/writing/Prensky\%20-\%20Digital\%20 Natives, \%20Digital \%20Immigrants $\% 20-\% 20$ Part1.pdf [Consultado el 11 de octubre de 2014]. 
. (2001b). "Do they really think differently?" En: On the Horizon, 9(5).

Recuperado de http://www.marcprensky. com/writing/Prensky\%20-\%20Digital\%20 Natives, \%20Digital\%20Immigrants\%20-\%20 Part2.pdf [Consultado el 11 de octubre de 2014].

Redecker, C. et al. (2011). En: The Future of Learning: Preparing for Change. Luxembourg: Publications Office of the European Union Comisión Europea- Institute for Prospective Technological Studies. Recuperado de http:// ftp.jrc.es/EURdoc/JRC66836.pdf [Consultado el 8 de febrero de 2014].

Rodríguez Juárez, C.; y Oxbrow, G. (2010). "Encouraging participation in online discussion forums with university EFL learners". En: Elia: Estudios de lingüística inglesa aplicada, 10, 137-166.

Sánchez Prieto, J.C.; Olmos Migueláñez, S. y García-Peñalvo, F.J. (2014).

"Understanding mobile learning: devices, pedagogical implications and research lines". En: Revista Teoría de la Educación: Educación y Cultura en la Sociedad de la Información, 15(1), 20-42. Recuperado de http://campus.usal.es/ revistas_trabajo/index. $\mathrm{php} / \mathrm{revistatesi/article/view/11651/12066}$ [Consultado el 29 de enero de 2015].
Seely Brown, J. (2005). "New Learning Environments for the 21st Century". En: Forum for the Future of Higher Education's 2005 Aspen Symposium. Recuperado de http:// www.johnseelybrown.com/newlearning.pdf [Consultado el 25 de enero de 2015].

Thomas, M.J.W. (2002). "Learning within incoherent structures: the space of online discussion forums". En: Journal of Computer Assisted Learning, 18, 351-366.

Torres Menárguez, A. (2014). “TOEFL o IELTS? Cómo elegir el mejor certificado de inglés". En: El País. Recuperado de http:// economia.elpais.com/economia/2014/12/08/ actualidad/1418035628_397560.html [Consultado el 10 de enero de 2015].

White, D.; y Le Cornu, A. (2011). "Visitors and Residents: A new typology for online engagement". En: First Monday, 16(9). Recuperado de http:/www.uic.edu/htbin/ cgiwrap/bin/ojs/index.php/fm/article/ viewArticle/3171/3049 [Consultado el 19 de octubre de 2014]. 


\section{Anexo: Cuestionario anónimo}

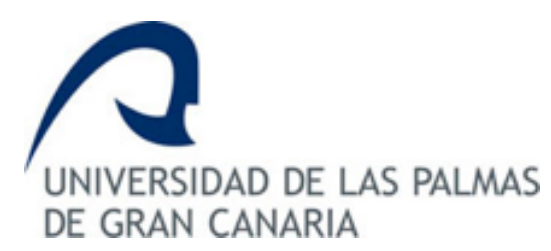

EFL/ESP and ubiquitous distance learning

Please answer the following questions. The feedback received will help us to identify priorities for improvements on u-learning research.

\section{You are:}

\begin{tabular}{|l|}
\hline$\square$ Man \\
$\square$ Woman \\
\hline
\end{tabular}

2. Age:

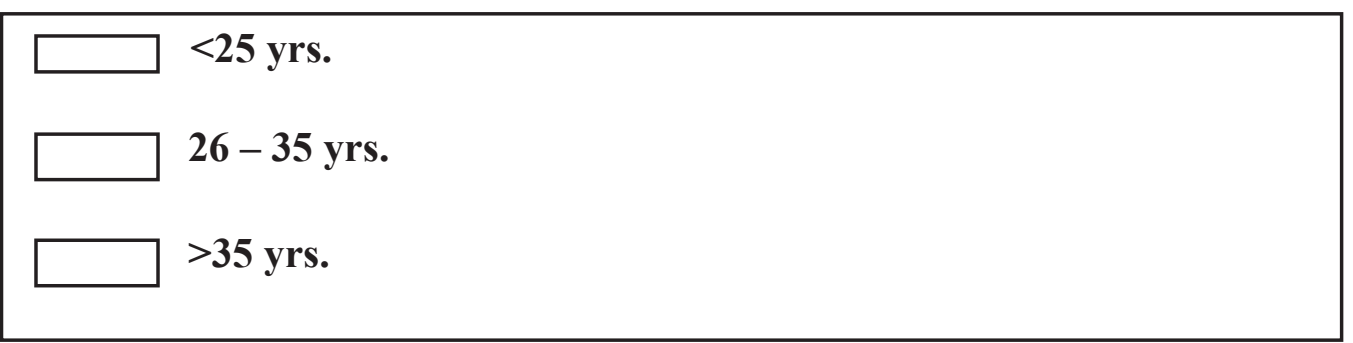

3. Your programme/subject name: Inglés (Trabajo Social)/Inglés (Educación Primaria):

4. Is the English Language subject you are studying optional $(\mathrm{O})$ or compulsory $(\mathrm{C})$ ?

5. Do you think that attendance is important for learning English? Yes No

6. What is the level of EFL used in your classes? Can you follow it?
Yes, always;
Yes, usually;
No, I can't
a. A1
c. B1
b. A2
d. B2 
7. Please circle the best alternative:
a. I have enjoyed the activities and work presented on this course: YES/NO
b. The structure of the online course was clear: YES/NO
c. The videos and online explanations helped me improve my learning skills: YES/ NO
d. Videos, PPT, and other online presentations have improved my reading/speaking/ listening/writing/grammar.
e. I prefer interactive activities that can be redone in the future: YES/NO
f. I ALWAYS/FREQUENTLY/RARELY/NEVER accessed the online tools provided.
g. I accessed THE VARIOUS PLATFORMS from MY COMPUTER AT HOME/
AT UNIVERSITY/ FROM MY MOBILE/ FROM MY IPAD/ FROM MY LAPTOP/ OTHER.
h. I also access other platforms that are open access (no registration or password is needed) to improve my English: YES/NO

8. Tick the $\mathbf{2}$ virtual learning environments that you have enjoyed the most. Write 1 for more enjoyable.
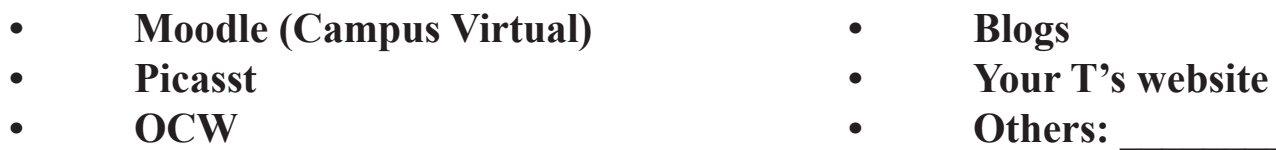

9. Which of the following is the activity you enjoyed most? Choose 3 examples and write 1 , for most enjoyable.
a. Discussion forums
b. Individual online presentations
using PPT or video formats (present.me, etc.)
c. Group online presentations using
PPT or video formats
d. Listening activities
e. Gap-fill exercises
f. Multiple choice exercises
g. Short-answer exercises
h. Individual reading exercises
i. Vocabulary exercises
j. Cooperative/Collaborative learning exercises (Glossaries, wikis, etc.)
k. Role-plays (speaking)
1. Others: Please, specify:

10. Would you say that your independent learning skills have been improved by means of e-learning activities? Yes No I don't know 
11. If you had to rate your English by means of these distance learning courses and tools, it would be?
1
2
3
4
5

Poor Good I don't know Very Good Excellent

12. If you had to rate this online communicative experience in English, it would be?
1
2
3
4
5

Poor Good I don't know Very Good Excellent

14. What aspects of the virtual platforms or of the English course you have done need to improve? (Any suggestion?)

THANK YOU VERY MUCH! WE APPRECIATE YOUR TIME!! 